\title{
A Personalized Recommendation Model for Tourism Products
}

\author{
Linqi Gao \\ Management School, Tianjin Normal University, Tianjin 300387, P.R. China \\ gaolqi@hotmail.com
}

\begin{abstract}
Electronic Commerce has becomes an important means for tourism enterprises to face the increasing competition. How to provide the personalized service for customers is an important issue to raise the service level of tourism. Through analyzing characteristics of tourism, a Personalized Recommendation Model is proposed at the basis of user's rating feature. It has following features: (1) Pre-processing user's rating data to solve different rating criterion of different user. (2) According to user's rating feature, a correction coefficient is set to ameliorate similarity among users, to improve the computational accuracy of the nearest neighbor. At last, experiments ware designed. Comparing with general collaborative filtering, the proposed algorithm has higher quality of recommendation.
\end{abstract}

Keywords: Business intelligence, Personalized service, Collaborative filtering, User classification, Electronic commerce

\section{INTRODUCTION}

With the development of Electronic Commerce, Personalized Recommendation System becomes an important research item [1]. From estimating the requirement of customer, Personalized Recommendation System actively proves the suitable product and services for individual.

At the same time, Electronic Commerce also becomes an important means for tourism enterprises [2]. Through advanced information technology tools, tourism eCommerce can improve internal and external connectivity of tourism enterprises to upgrade the management level and integrating ability of tourism resources, and contribute to the achievement of personalized demand of consumer.

In this article, characteristics of tourism are analyzed, firstly. Then, a Personalized Recommendation Model is proposed to provide exact recommendation at the basis of user's rating behavior. It has following steps: (1) Pre-processing user's rating data to solve different rating criterion of different user, (2) According to user's rating feature, a correction coefficient is set to ameliorate similarity among users, to improve the computational accuracy of the nearest neighbor. (3) Current user's attitude is forecast at the basis of neighbors' rating data and recommendation is produced.

Please use the following format when citing this chapter:

Gao, L., 2007, in IFIP International Federation for Information Processing, Volume 255, Research and Practical Issues of Enterprise Information Systems II Volume 2, eds. L. Xu, Tjoa A., Chaudhry S. (Boston: Springer), pp. 1401-1405. 


\section{PERSONALIZED RECOMMENDATION MODEL}

\subsection{General Collaborative Filtering Recommendation}

The collaborative filtering (CF) recommendation acts according to other users' viewpoint to produce recommendation to the goal user. Its basic thought is based on a supposition: If user grade to some product quite similarly, then they grade to other product also similar [3-5]. CF recommendation model use statistics technology to search goal user's recent neighbors, then forecast goal user's grading according to neighbors' grades, and thus has the recommendation.

The rating data of customer forms an $m \times n$ matrix, A (m, $\mathrm{n})$. In the matrix, row $m$ represents customer $m$, column $n$ represents item $n$. The element $R_{i, \mathrm{j}}$ in $i^{\text {th }}$ row and $j^{\text {th }}$ column represents the rating data of customer $i$ on item $j$. To find user's neighbors, the similarity among users must be measured, represented as $\operatorname{sim}(i, j)$.

The precise computation of goal user's neighbors is the successful key of CF model. It is necessary to attempt new algorithm foundation to increase the recommendation precision.

In general CF model, identical algorithm is used to produce recommendation about all users. But in practical application, it is not realistic. In the following, a novel CF model is proposed at the basis of user's rating habit

\subsection{CF Model Based on User's Rating Habit}

Each user's rating data has a domain: $[\min , \max ]$, respectively indicate the lowest and the most value of current user. The goal of classification is to find users having similar rating characteristic, then forecasting user's preference. The user classification method is shown in Figure 1.

The "average" is average score of user's rating data. The "max" and "min" respectively express the lowest and the most value of the user. $\alpha$ and $\beta$ are two parameters to classify each average. $\lambda$ is a parameter to describe distance between $\max$ and $\min$. And $\tau$ is a threshold to restrict " $n p_{h}$ " and " $n p_{l}$ ".

" $n p_{h}$ " expresses the ratio of number of item having high score about all rated item, namely:

$$
n p_{h}=\frac{\text { number of items rated highly }}{\text { number of items rated }}
$$

" $n p_{l}$ " expresses the ratio of number of item having low score about all rated item, namely:

$$
n p_{l}=\frac{\text { number of items rated lowly }}{\text { number of items rated }}
$$


Therefore, all users are divided into three kinds: (1) User which always giving average score, (2) User which giving high score to minority item, (3) User which giving low score to minority item, respectively marked with Type I, Type II, Type III.

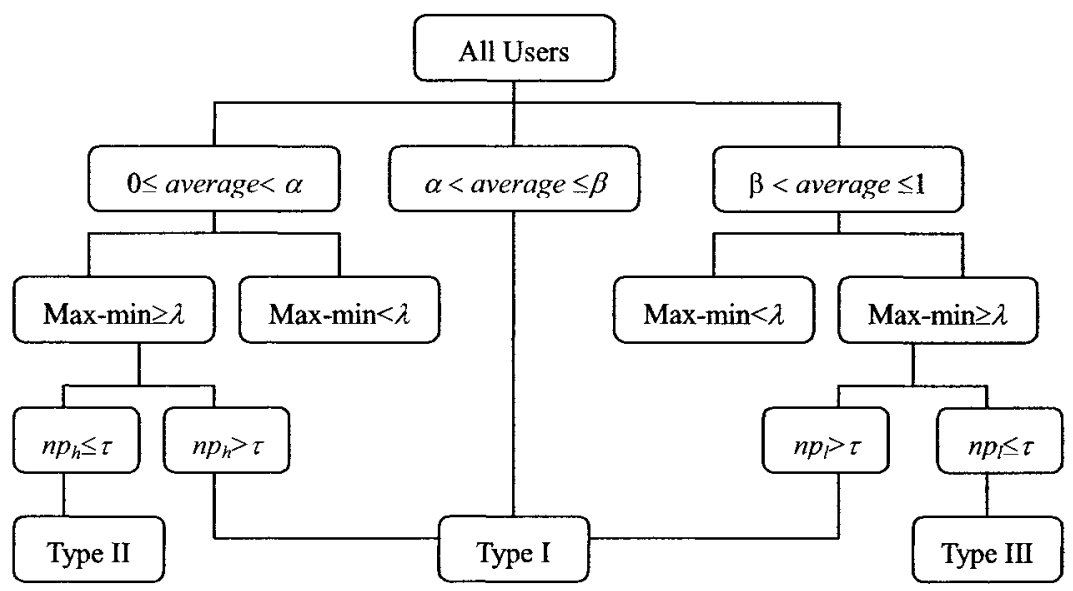

Figure 1. User Classification

There are many kinds of methods to compute similarity among users $[6,7]$, here, and cosine similarity is used. According to user's type, similarity is revised by corresponding coefficient, described in table 1 .

Table 1. Coefficient Table

\begin{tabular}{|l|l|l|l|}
\hline & Type I & Type II & Type III \\
\hline Type I & 1 & 0.5 & 0.5 \\
\hline Type II & 0.5 & 1 & 0 \\
\hline Type III & 0.5 & 0 & 1 \\
\hline
\end{tabular}

\subsection{Recommendation Produced}

According to classified result, the precise similarity can be obtained. Current customer's rating data can be forecasted at the basis of neighbors' average rating. Then recommendation can be gotten.

Supposes neighbors set of current customer $u$ is $N_{u}$, the forecasted rating $P_{u, i}$ of customer $u$ about item $i$ is

$$
P_{u, i}=\bar{R}+\frac{\sum_{n \in N_{u}} c(u, n) \operatorname{sim}(u, n)\left(R_{n, i}-\overline{R_{n}}\right)}{\sum_{n \in N_{u}} \operatorname{sim}(u, n)}
$$


$\operatorname{sim}(u, n)$ expresses similarity between usre $u$ and $n, c(u, n)$ expresses revising coefficient according to kind of user $u$ and $n, R_{n, i}$ expresses rating of customer $n$ about item $i$, both users' average rating is applied as the rating basis. When computing user's average score about an item, only rated items are involved. It means that sum of user's rating data divide number of non-zero item, then user's average rating score is gotten.

\section{EXPERIMENT AND ITS ANALYSIS}

Experimental data are selected from a traveling e-Commerce Website to design experiment, randomly select 3,000 terms as the experimental data set, contains 92 users and 30 traveling items.

Mean Absolute Error (MAE) is applied to evaluate recommendation quality. Let forecasted rating set is $\left\{p_{1}, p_{2}, \ldots, p_{n}\right\}$, the real rating set is $\left\{q_{1}, q_{2}, \ldots, q_{n}\right\}$, then

$$
M A E=\frac{\sum_{i=1}^{n}\left|p_{i}-q_{i}\right|}{n}
$$

Fig. 2 shows the result of comparison. The experimental results show the proposed method has higher capability.

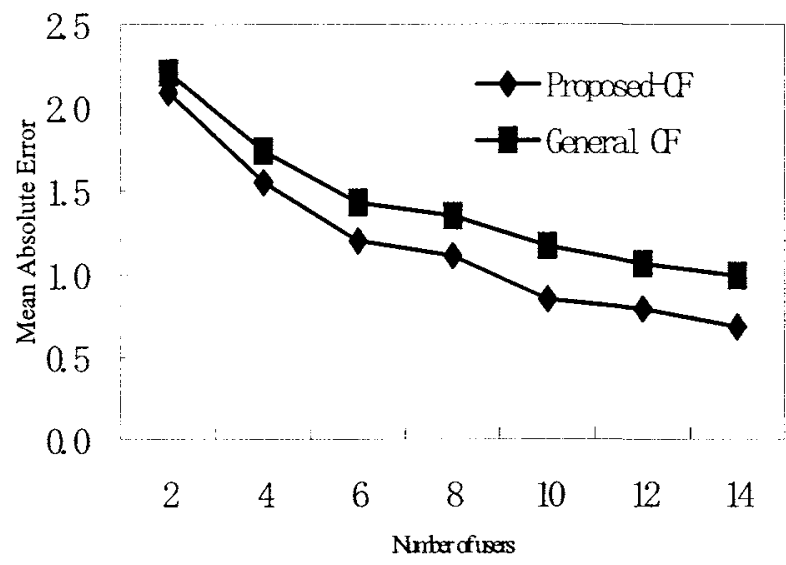

Figure 2. Comparison Between Proposed-CF and General CF 


\section{CONCLUSIONS}

The classifying of customer is the basis of recommendation algorithm, it is necessary through the analyzing feature of application field to design classifying algorithm. According to user's rating feature, a Personalized Recommendation Model is proposed. It has following features: (1) Pre-processing user's rating data to solve different rating criterion of different user. (2) According to user's rating feature, a correction coefficient is set to ameliorate similarity among users, to improve the computational accuracy of the nearest neighbor. Experiments designed show the proposed algorithm has higher quality of recommendation than general CF model.

In the future, more experiment will be done to examine reliability of the proposed model and speed. More classifying method about user's rating feature will also be applied to decide the best method.

\section{ACKNOWLEDGEMENTS}

The Research is Supported by (1) the National Science Fund of China under Grant No. 70402009; (2) University science and technology development fund of Tianjin Education Committee under Grant No. 20030613; (3) Doctor Research Fund of Tianjin Normal University Grant No. 52RL005.

\section{REFERENCES}

1. R. Bergmann and P. Cunningham, Acquiring Customer's Requirement in Electronic Commerce, Artificial Intelligence Review. Volume 18, Number 2, pp.163-169, (2002).

2. W. Guo and L. Gao, Research of Personalized Information Service Pattern Of Electronic Tourism Intermediates, Informatics Science. Volume 5, Number 4, pp.732-735, (2006).

3. Y.H. Cho and J.K. Kim, Application of Web usage mining and product taxonomy to collaborative recommendations in e-commerce, Expert Systems with Application. Volume 26, Number 2, pp.233-246, (2004)

4. .K.W. Cheung, Learning user similarity and rating style for collaborative recommendation, Information Retrieval. Volume 7, Number 3, pp. 395-410, (2004).

5. .L. Gao and L. Li, Production recommender method based on customer's behavior, Computer Engineering and Application. Volume 41, Number 3, pp.188-190, (2005).

6. G. Adomavicius, Toward the next generation of recommender systems: a survey of the state-of-the-art and possible extensions, IEEE Transactions on Knowledge and Data Engineering. Volume 17, Number 16, pp.734-749, (2005)

7. J. Herlocker and J.A. Konstan, An Empirical Analysis of Design Choices in Neighborhood-Based Collaborative Filtering Algorithms, Information Retrieval. Volume 5, Number 2, pp.287-310, (2002). 